\title{
Superior Mesenteric Artery Aneurysm Presenting with Chronic Abdominal Pain: A Case Report
}

\author{
Mohammad Enamul Hakim ${ }^{1}$, MIS AI-Manzo ${ }^{1}$, F Aaysha Cader ${ }^{2}$, Naresh Chandra ${ }^{1}$, Fidah Hossain ${ }^{1}$ \\ ${ }^{1}$ Department of Vascular Surgery, NICVD, Dhaka, ${ }^{2}$ Department of Cardiology, NICVD, Dhaka.
}

\author{
Key words: \\ Superior \\ mesenteric artery \\ aneurysm, aorto- \\ mesenteric \\ bypass, PTFE \\ graft
}

\begin{abstract}
:
Superior mesenteric artery (SMA) aneurysm is a rare vascular entity and may present with a wide range of symptoms. They are often symptomatic, presenting with thromboembolic intestinal angina. They have a high risk of rupture and may present with gastro-intestinal bleeding, associated with high morbidity and mortality. We report the case of a 14year-old boy who presented with chronic abdominal pain and weight loss, in which SMA aneurysm was diagnosed after radiological evaluation. He underwent successful excision of aneurysmal sac along with aorto-mesenteric bypass using polytetrafluoroethylene (PTFE) graft, and had an uneventful recovery.
\end{abstract}

(Cardiovasc. j. 2016; 8(2): 161-164)

\section{Introduction:}

Visceral artery aneurysms (VAAs) are a rare entity in clinical practice, with a reported incidence ranging from $0.1 \%$ to $2 \% .{ }^{1}$ Splenic and hepatic artery aneurysms account for the majority of VAAs (60\% and $20 \%$, respectively). Although only about $5.5-6.9 \%$ of VAAs are attributable to superior mesenteric artery (SMA) aneurysms, ${ }^{2-4}$ they are clinically important due to severity of complications, including thrombosis, embolism and rupture, leading to mesenteric ischaemia and fatal haemorrhage, even death (up to $40 \%$ ). ${ }^{4,5}$ There is no gender predilection for SMA aneurysms. ${ }^{4,6}$ They most commonly occur in the proximal $5 \mathrm{~cm}$ of the artery. ${ }^{2,4,6}$ Formerly, infection was considered the most common cause of SMA aneurysm, with the majority of patients presenting with fever, abdominal pain, and a pulsatile abdominal mass. ${ }^{7,8}$

In the majority of cases, SMA aneurysms are of mycotic etiology (60\%), with atherosclerotic ones being less frequent, and other causes including medial degeneration, connective tissue disease and trauma. ${ }^{9-11}$ VAAs are now being diagnosed with increasing frequency, with the advent of increased availability and augmented use of computed tomography (CT), Magnetic Resonance Imaging (MRI) and other cross-sectional imaging. More than $90 \%$ of SMA aneurysms are symptomatic, usually presenting with abdominal pain and gastrointestinal bleeding. ${ }^{9-}$ ${ }^{11}$ Furthermore, acute mesenteric ischemia may result from thromboembolism arising from the aneurysm. ${ }^{4} \mathrm{~A}$ small number of patients with SMA aneurysms may remain asymptomatic. ${ }^{11}$ Typically, surgery or endovascular management is considered for VAAs when detected in order to prevent rupture and subsequent mortality. ${ }^{4,11} \mathrm{We}$ report this case of teenage Bangladeshi male who presented with a large pulsatile mass mimicking an abdominal aortic aneurysm (AAA). Radiological investigations revealed a large SMA aneurysm, and he underwent successful excision of aneurysmal sac along with aorto-mesenteric bypass.

\section{Case Report}

A 14 year old teenage male presented with intermittent abdominal pain for 2 years; pain was mostly localized to the epigastric region with no radiation, commonly occurred immediately postprandially, and was relieved by vomiting; vomitus comprised of non-bilious ingested food particles. He also admitted to an extreme fear of taking food due to the subsequent severe abdominal pain that ensued post-prandially, thus resulting in significant weight loss of approximately $15 \mathrm{~kg}$ over the duration of the illness. There was no prior history

Address of correspondence: Dr. Mohammad Enamul Hakim, Department of Vascular Surgery, National Institute of Cardiovascular Disease, Dhaka, Bangladesh. Email: drmehakim@yahoo.com 
of trauma, fever or gastro-intestinal blood loss. He was a non-smoker, and not known to be hypertensive or diabetic. On examination, he was mildly anaemic and cachectic with stable haemodynamic parameters.Abdominal examination revealed a pulsatile, non-tender mass with illdefined margins measuring approximately $3 \mathrm{~cm} x$ $2 \mathrm{~cm}$ in the epigastric region; it was intra-abdominal \& freely mobile from side to side but restricted from above downwards.

Initially ultrasonography detected evidence of an abdominal aortic aneurysm. On further detailed evaluation by $\mathrm{CT}$ angiography of the aorta and its branches, the aneurysm was found to be originating from the superior mesenteric artery. A saccular dilatation of the mid SMA was noted, with the dilated segment measuring $3.9 \mathrm{~cm} \times 2.3$ $\mathrm{cm}$ along with thrombus. Large irregular calcification was noted in lower part of thrombus, in the region of SMA.

Following adequate preperation, surgery was done: the aorta was explored via intra peritoneal approach \& SMA was identified at its origin; its distal part was explored along the gut. Aneurysmal sac was excised and bypass grafting was done between infra-renal part of aorta and SMA distal to the aneurysm, using polytetrafluoroethylene (PTFE) graft. Gastro-intestinal activity resumed after 2 days and oral nutrition was commenced 3 days after surgery. The patient was discharged from the hospital on $5^{\text {th }}$ post-operative day in stable condition and has been well and symptom-free at subsequent follow up.

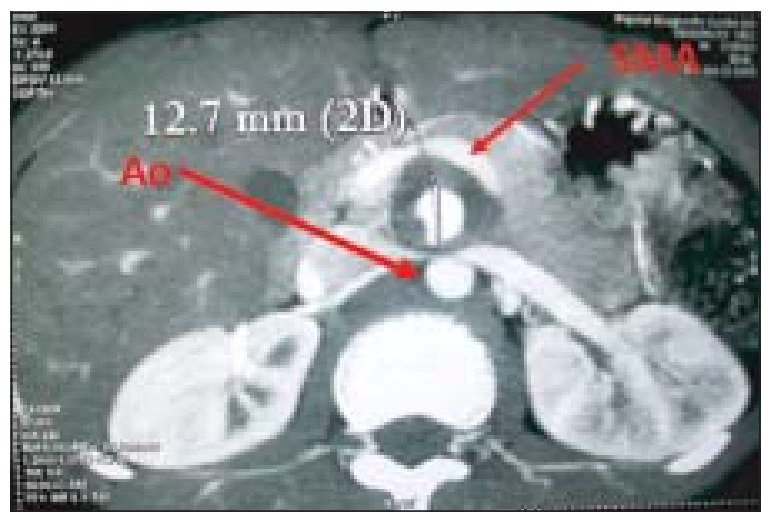

Fig.-1: CT scan showing a saccular dilatation of the mid superior mesenteric artery, with the dilated segment measuring $3.9 \mathrm{~cm} \times 2.3 \mathrm{~cm}$ along with thrombus.

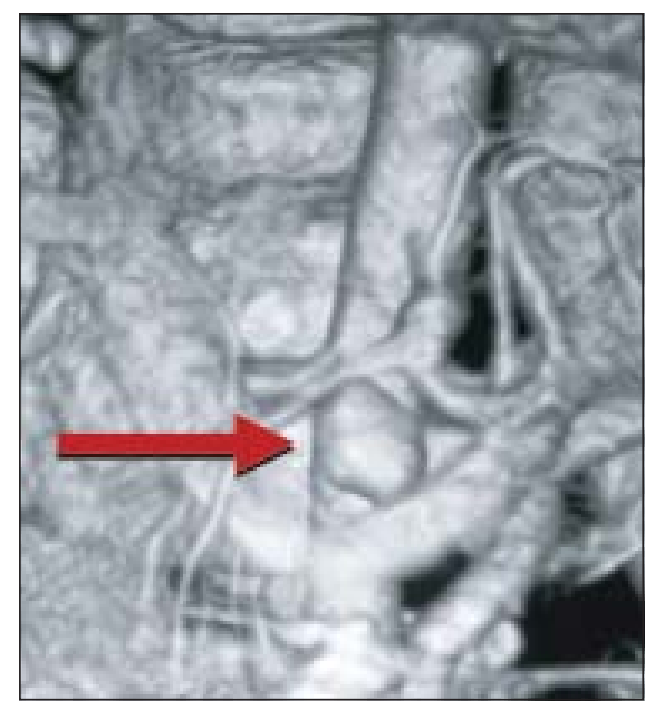

Fig.-2: $C T$ scan (3D reconstruction) showing a saccular dilatation of the superior mesenteric artery. The coeliac trunk appears normal.

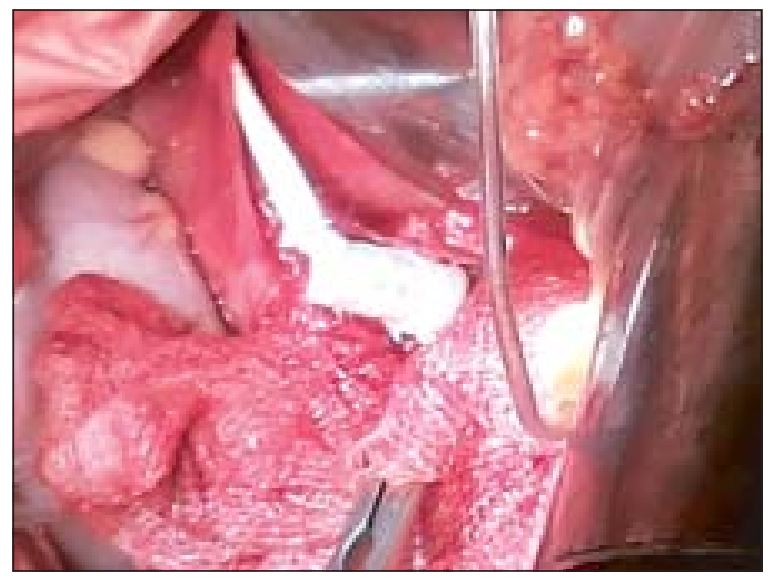

Fig.-3: On-table image during surgery showing 7 $\mathrm{mm}$ ringed graft which was anastomosed with the aorta, the distal end of which was anastomosed to a $4 \mathrm{~mm}$ non-ringed graft. The latter was then anastomosed to SMA.

\section{Discussion:}

Reports of visceral artery aneurysms date back to more than 230 years. ${ }^{12}$ Stevenson attempted the first surgical repair of a SMA aneurysm in 1895, which unfortunately resulted in death from hemorrhage. ${ }^{13}$ It was not until 1949 that DeBakey and Cooley performed the first successful resection of a mycotic SMA aneurysm secondary to bacterial endocarditis in a young female. ${ }^{14}$ Since then, the understanding of the pathophysiology, natural history, diagnosis, and treatment has improved but 
is tempered by the relative rarity of visceral artery aneurysms.

SMA aneurysms are usually located within the first $5 \mathrm{~cm}$ from the origin of the SMA and can be fusiform or saccular. ${ }^{2,4}$ In contrast to most other aneurysms, the prevalence is equally distributed among males and females, although males are reported to have a higher risk of rupture. ${ }^{4}$ There is no typical age of onset of SMA aneurysms, which depends primarily on the aetiology of the disease. Most patients with mycotic aneurysms are younger than 50 years of age, and patients with atherosclerotic aneurysms are usually in their seventh or eighth decade. ${ }^{15}$

In comparison to all other visceral aneurysms, 70$90 \%$, of SMA aneurysms are symptomatic at presentation. ${ }^{11,16}$ The majority of patients present with significant and progressive abdominal pain, with some of them developing typical intestinal angina with postprandial pain..$^{9,11}$ In many cases it is difficult to determine if the aetiology is due to relative mesenteric ischemia or to direct aneurysm expansion. A mobile, tender, pulsatile mass is often seen, as are symptoms such as nausea, vomiting, and gastrointestinal bleeding. ${ }^{9}$ Fever may be a prominent finding in mycotic aneurysms. ${ }^{17}$

The natural history of SMA aneurysm appears to be one of expansion and eventual rupture. ${ }^{18}$ Other complications of SMA aneurysms include thrombosis and distal embolization causing intestinal ischemia, and erosion into adjacent bowel with gastrointestinal bleeding. Up to $38-50 \%$ of patients present with a ruptured aneurysm. ${ }^{2,4,19}$ The resultant mortality rate (between 30 to $90 \%$ ) is significantly worse than in those patients undergoing repair of intact aneurysms $(<15 \%)$. 9,20 The indications for repair include SMA aneurysms with a diameter $\geq 2 \mathrm{~cm}$, symptomatic SMA aneurysms, and rapidly growing SMA aneurysms. ${ }^{21}$ However, as the natural history of SMA aneurysms is not well-defined, there is still some controversy about the absolute indications for their repair and surgical intervention. ${ }^{11}$ Some surgeons prefer repair irrespective of symptom, due to the high incidence of rupture and its associated mortality.

Open repair is still considered the "gold standard" for all SMAAs in patients who require treatment. Open surgical repair can be performed by surgical resection with arterial reconstruction, as in our case, or by ligation of the vessel without reconstruction. The primary goal of therapy is to exclude the aneurysmal sac from the blood circulation. Ligation without reconstruction should only be performed in cases in which sufficient collaterals are present to provide collateral flow. ${ }^{22}$ Temporary SMA occlusion and observation of the small bowel viability is recommended. Arterial reconstruction of the SMA after ligation of the aneurysm can be performed with an interposition graft if the viability of the small bowel is suspected. ${ }^{22}$ Due to high incidence of infectious aetiology; saphenous grafts are preferred over prosthetic grafts. ${ }^{22}$

Endovascular modalities may also be considered in these patients; they are associated with lower morbidity and mortality and shorter hospital stay. However, as it is a technically difficult approach, requiring specific training and an initial learning curve, they are not the preferred modality in case of many aneurysms. ${ }^{23,24}$ Endovascular treatment is primarily used in small asymptomatic aneurysms in anatomical locations where surgery is potentially difficult or dangerous. The treatment choices include embolization of the aneurysm or stent grafting. Anatomy conducive to transcatheter embolization includes aneurysms with a small neck, which are distal to the origin of the SMA and assessment of bowel viability should be warranted with angiographic determination of collateral flow. ${ }^{4}$

\section{Conclusion:}

Despite significant advances in treatment modalities, delayed detection leads to SMA aneurysms still remaining untreated, leading to serious and life-threatening consequences. A high degree of clinical suspicion is required in order to diagnose SMA aneurysms early and institute prompt surgical or endovascular treatment before the onset of fatal complications. Acceptable outcomes can only be achieved with timely diagnosis and intervention prior to aneurysm rupture. Apart from open surgical modality, endovascular embolization provides an additional therapeutic option to those high risk candidates with favourable aneurysm anatomy.

Conflict of Interest - None. 


\section{References:}

1. Wojtaszek M. Managing Visceral Artery Aneurysms. Current endovascular techniques and technologies for treating this increasingly encountered presentation. Endovascular Today (serial online) 2013 Oct:77-81.

2. Messina LM, Shanley CJ. Visceral artery aneurysms. Surg Clin North Am 1997;77(2):425- 442.

3. Carr SC, Pearce WH, Vogelzang RL, McCarthy WJ, Nemcek AA Jr., Yao JS. Current management of visceral artery aneurysms. Surgery 1996;120(4):627633.

4. Stone WM, Abbas M, Cherry KJ, Fowl RJ, Gloviczki P. Superior mesenteric artery aneurysms: Is presence an indication for intervention? J Vasc Surg 2002;36(2): $234-237$.

5. Dasari BV, Mullan M, Lau L, Loan W, Lee B. A 6.5-cm pseudoaneurysm of the superior mesenteric artery managed by primary surgical repair. Vascular 2013;21(1):39 -42.

6. Jiang J, Ding X, Su Q, Zhang G, Wang Q, Jian W, Wang $\mathrm{Z}, \mathrm{Hu}$ S. Therapeutic management of superior mesenteric artery aneurysms. J Vasc Surg 2011;53(6):1619-1624.

7. McNamara MF, Griska LB. Superior mesenteric artery branch aneurysms. Surgery 1980;88:625-630.

8. Verma BS, Bose AK, Bhatia HC, Katoch R. Superior mesenteric artery branch aneurysm diagnosed by ultrasound. Brit J Radiol 1991; 64:169 -171.

9. Pulcini G, D’Adda F, Lanzi S, Giampaoli F, Pouchè A. Aneurysms of the superior mesenteric artery. Ann Ital Chir 2002;73(2):129-136.

10. Pitton MB, Dappa E, Jungmann F, Kloeckner R, Schotten S, Wirth GM, et al. Visceral artery aneurysms: Incidence, management, and outcome analysis in a tertiary care center over one decade. Eur Radiol 2015; 25(7): 2004-2014.

11. Badea R, Barsan M, Scridon T, Miclaus G, Pascu O, Molnar G, et al. Superior Mesenteric Artery Aneurysm: Importance of Sonography as the Primary Imaging Technique for Detection. J Ultras Med 2010;29(10): 1503-1506.

12. Beaussier M: Sur unanevrisme de l'arteresplinque don't les parois se sontossifiees. J Medical Toulose 1770;32:157.
13. Stevenson WF. Case of abdominal aneurysm treated by laparotomy and introduction of wire into sac with death. Lancet 1985;1:22-23.

14. DeBakey ME, Cooley DA: Successful resection of mycotic aneurysm of superior mesenteric artery: Case report and review of literature. Am Surg 1953;19:202-212.

15. Kopatsis A, D'Anna JA, Sithian N, Sabido F. Superior mesenteric artery aneurysm: 45 years later. Am Surg 1998;64:263-266.

16. Wagner WH, Allins AD, Treiman RL, Cohen JL, Foran RF, Levin PM, et al.Ruptured visceral artery aneurysms. Ann Vasc Surg 1997;11(4):342- 347.

17. Lorelli DR, Cambria RA, Seabrook GR, Towne JB. Diagnosis and management of aneurysms involving the superior mesenteric artery and its branches-a report of four cases. Vasc Endovascular Surg 2003;37(1):59-66.

18. Carr SC, Pearce WH, Vogelzang RL, McCarthy WJ, Nemcek AA Jr, Yao JS. Current management of visceral artery aneurysms. Surgery 1996;120(4):627-634.

19. Lindberg CG, Stridbeck H: Aneurysms of the superior mesenteric artery and its branches. Gastrointest Radiol 1992;17:132-134.

20. Settembrini PG, Jausseran JM, Roveri S, Ferdani M, Carmo M, Rudondy P, et al. Aneurysms of anomalous splenomesenteric trunk: Clinical features and surgical management in two cases. J Vasc Surg 1996; 24(4): 687-692.

21. Chadha M, Ahuja C. Visceral Artery Aneurysms: Diagnosis and Percutaneous Management. Semin Intervent Rad 2009; 26(3): 196-206.

22. Komori K, Mori E, Yamaoka T, Ohta S, Takeuchi K, Matsumoto T, et al. Successful resection of superior mesenteric artery aneurysm: A case report and review of theliterature. J Cardiovasc Surg (Torino). 2000; 41(3):475-478.

23. Mendonça CT, Weingartner J, de Carvalho CA, Costa DS. Endovascular treatment of contained rupture of a superior mesenteric artery aneurysm resulting from neurofibromatosis type I. J Vasc Surg 2010;51(2):461464.

24. Ruiz-Tovar J, Martínez-Molina E, Morales V, Sanjuanbenito A, Lobo E. Evolution of the therapeutic approach of visceral artery aneurysms. Scand J Surg 2007;96(4):308-313. 